\title{
Shock due to urosepsis: A multicentre study
}

\author{
Fukashi Yamamichi, MD; ${ }^{1}$ Katsumi Shigemura, MD;1,2,3 Koichi Kitagawa, MT; Kei Takaba, MT;5 \\ Issei Tokimatsu, MD, ${ }^{3}$ Soichi Arakawa, MD, ${ }^{1,3}$ Masato Fujisawa, MD'
}

\begin{abstract}
'Division of Urology, Department of Organ Therapeutics, Kobe University Graduate School of Medicine, Kobe, Japan; ${ }^{2}$ Department of International Health Sciences, Kobe, Japan; ${ }^{3}$ Infecion Control and

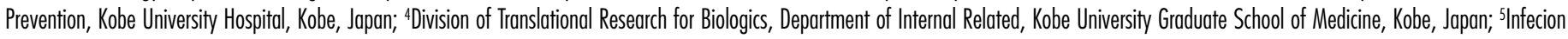
Control and Prevention, Mie Prefectural General Medical Centre, Yokkaichi, Japan
\end{abstract}

Cite as: Can Urol Assoc J 2017;11 (3-4):E105-9. http://dx.doi.org/10.5489/cuaj.4097 Published online March 16, 2017

\section{Abstract}

Introduction: Urosepsis is a severe infection that can cause shock afterwards. The purpose of this study is to investigate the clinical and bacterial risk factors for shock in those cases with urosepsis caused by urinary tract infection in a multicentre study.

Methods: Our study included 77 consecutive urosepsis cases from four hospitals. We examined factors such as patient characteristics, underlying disease, serum white blood cell (WBC) count, platelet count, C-reactive protein (CRP) level at the time of diagnosis of urosepsis, urinary tract occlusion, causative bacteria, and bacterial antibiotic susceptibilities. Statistical analyses were performed to assess the potential risk factors for shock during the clinical course of urosepsis by a multivariate analysis.

Results: We had 38 male and 39 female patients aged 25-104 (median 73). Underlying diseases included cancers $(n=22,28.6$ $\%$ ) and diabetes mellitus ( $\mathrm{n}=17,22.1 \%)$. Positive blood culture was seen in 74 cases; these involved 88 bacterial strains, of which Escherichia coli was the most common (34 strains, $38.6 \%$ ). There were 31 cases with shock (40.3\%) and multivariate analyses demonstrated that serum CRP was the only clinical risk factor for shock due to urosepsis.

Conclusions: Our study demonstrated that serum CRP was a risk factor for shock during urosepsis in a multicentre analysis. Further prospective studies with a greater number of patients are needed to draw more definitive conclusions.

\section{Introduction}

Urinary tract infection (UTI) is a frequent cause of bacteremia and we have seen many cases in which antibiotic therapies were ineffective, partly owing to the spread of attenuated bacteria and increase of antibiotic-resistant strains. ${ }^{1}$ UTI is generally classified as uncomplicated or complicated according to the presence of urinary tract and/or systematic underlying diseases. ${ }^{2}$ Complicated UTI, in particular, sometimes causes or leads to urosepsis, defined as bacteremia associated with urinary tract occlusion by stone or cancer, for instance. ${ }^{3,4}$ In addition, urosepsis are often related to systematic diseases that compromise the immune system, such as diabetic mellitus (DM) or steroid-dosing. ${ }^{2}$

Urosepsis patients can go into fatal shock, ${ }^{5}$ therefore, rapid and accurate diagnosis and treatment are vital, along with an association of the risk factors for shock. ${ }^{6}$ The risk factors for shock in urosepsis have not been widely studied, especially not in a multicentre setting, where a variety of patient characteristics exist and results may differ from hospital to hospital. ${ }^{2}$ We undertook this multicentre study to explore the risk factors for shock during urosepsis in patients with UTI or urological infections such as kidney or retroperitoneal abscess.

\section{Methods}

The data were gathered and reviewed from Mie Prefectural General Medical Centre, Kobe University Hospital, Kobe City Hospital Organization; Kobe City Medical Centre West Hospital, and Hyogo Prefectural Amagasaki Medical Centre. We retrospectively investigated the risk factors for shock due to urosepsis in 77 patients hospitalized in urology wards. This study was approved by the institutional review board (IRB) of Kobe University (IRB No. 1872). All procedures performed in studies involving human participants were in accordance with the ethical standards of the institutional research committee and with the 1964 Helsinki declaration.

We examined as potential risk factors for shock due to urosepsis, the patient characteristics, including underlying diseases (such as malignancy or DM); laboratory tests at the time of diagnosis of urosepsis, ${ }^{7}$ such as serum white blood cell (WBC) count, platelet count, C-reactive protein (CRP) levels, urinary tract occlusion, and causative bacteria isolated from blood and their antibiotic susceptibilities (Table 1).

All patients were tested for causative bacteria from the abscess, urine, or blood.

Susceptibility testing was performed by measuring minimal inhibitory concentration (MIC) for several kinds of antibiotics: ampicillin (ABPC), piperacillin (PIPC), ampicillin/ 
Yamamichi et al.

sulbactam (ABPC/SBT), cefazolin (CEZ), ceftazidime (CAZ), cefepime (CFPM), cefmetazole (CMZ), aztreonam (AZT), imipenem (IPM), meropenem (MEPM), minocycline (MINO), amikacin (AMK), gentamicin (GM), ciprofloxacin (CPFX), and levofloxacin (LVFX). The definition of susceptibility and the ranges of concentrations tested were based on 2008 Clinical Laboratory Standards Institute (CLSI) guideline M07A8 (Table M100-S19), using Frozen plates (Eiken Chemical Co. Ltd., Tokyo, Japan).

Septic shock was defined by general standards according to the American College of Chest Physicians/Society of Critical Care Medicine ACCP/SCCM definition ${ }^{8}$ and shock was diagnosed during the course of urosepsis until confirmation of cure.

Urosepsis was defined as sepsis caused by infection of the urinary tract and/or male genital organs. ${ }^{4}$ Septic shock is severe sepsis plus a state of acute circulatory failure characterized by persistent arterial hypotension (defined as a systolic arterial pressure below $90 \mathrm{mmHg}$, a mean arterial pressure $<60 \mathrm{mmHg}$, or a reduction in systolic blood pressure of $>40 \mathrm{mmHg}$ from baseline) unexplained by other causes and despite adequate volume resuscitation. ${ }^{9}$

As a statistical analysis, chi-square tests were performed to correlate patient characteristics, such as gender or presence of urinary tract occlusion, and shock. A Student's t-test was used to determine the association between age or blood tests and shock, and logistic regression tests were conducted to detect the risk factors for shock.

\section{Results}

Patient distribution included 25 cases from Mie Prefectural General Medical Centre, 25 cases from Kobe University Hospital, 23 cases from Kobe Municipal Medical Centre West Hospital, and four cases from Hyogo Prefectural Amagasaki General Medical Centre. Patient characteristics are shown in Table 1 . Thirty-one cases (40.3\%) had shock. Gender distribution was 38 male and 39 female aged 25-104 years (median 73). All the cases were complicated UTI with urinary tract underlying diseases. General underly-

\begin{tabular}{lcccc}
\hline \multicolumn{5}{l}{ Table 1. Patient characteristics } \\
\hline & $\mathbf{n}$ & Shock & No shock & $\mathbf{p}$ \\
\hline Male & 38 & 10 & 28 & \\
Female & 39 & 21 & 18 & \\
Total & & 31 & 46 & $<\mathbf{0 . 0 0 0 1}$ \\
Age, range, median & $25-104$ & $41-89$ & $25-104$ & 0.3775 \\
& $(73)$ & $(75)$ & $(72)$ & \\
Underlying diseases & & & & \\
$\quad$ Cancer & 22 & 4 & 18 & \\
$\quad$ Diabetes mellitus & 17 & 8 & 9 & \\
$\quad$ Neurological disease & 11 & 4 & 7 & 0.1497 \\
\hline
\end{tabular}

ing diseases included 22 malignancies (bladder cancer: 16 cases; prostate cancer: two cases; uterine cancer: two cases), $17 \mathrm{DM}$, and 11 brain diseases. Significantly more females experienced shock $(p<0.0001)$ (Table 1).

There were 55 cases with urinary tract occlusion. The details of drainage are shown in Table 2 . There were significantly more cases of shock with urinary tract occlusion than without occlusion $(p<0.0001)$. There was no significant difference related to the kind of stent, but intervention, such as stenting or nephrostomy, was necessary in more shock cases compared to no-shock cases $(\mathrm{p}=0.003)$ (Table 2$)$.

The laboratory data are shown in Table 3. Serum WBC was $3100-54670 / \mu \mathrm{L}$ (median $14400 / \mu \mathrm{L}$ ), platelets $1.3-149.9 \times 10^{4} / \mu \mathrm{L}$ (median $15.5 \times 10^{4} / \mu \mathrm{L}$ ), CRP 0.284-47.9 $\mathrm{mg} / \mathrm{dL}$ (median $8.603 \mathrm{mg} / \mathrm{dL}$ ). The shock cases had significantly higher CRP than non-shock cases $(\mathrm{p}=0.003)$ (Table 3 ).

Seventy-five (97.4\%) cases (89 bacteria) were positive for bacteria. Escherichia coli (E. coli) was the most often seen (34 cases, $38.2 \%$ ) and eight of 34 isolates $(23.5 \%)$ were extended spectrum beta-lactamase (ESBL) producers. (Table 4). The $E$. coli most often isolated in this study are shown in Table 5. Five of 34 isolates (14.7\%) were ESBL producers and fluoroquinolone-resistant.

The antimicrobial agents used are shown in Table 6 . In those cases with shock, MEPM was used most often (51.6\%) and in cases without shock, tazobactam/piperacillin (TAZ/ PIPC) was used most often (21.7\%); importantly, there was a significant difference in the use of antimicrobial agents between the cases with shock and without shock $(p=0.0324)$.

Our multivariate analysis of risk factors for shock demonstrated that higher CRP was an independent risk factors for shock due to urosepsis $(p=0.0041)$ (Table 7).

\section{Discussion}

Urosepsis generally occurs in 15-30 \% of acute pyelonephritis cases ${ }^{10}$ and UTI is one of the leading causes (severe sepsis $9 \%$ and septic shock $31 \%$ ). ${ }^{11}$ Unfortunately, in 20-40 $\%$ of cases, uroseptic shock leads to death. ${ }^{4}$ Urosepsis needs to be understood as a severe condition, especially in the cases involving immunocompromised hosts or urinary tract

\section{Table 2. Urinary tract occlusion and drainage}

\begin{tabular}{lcccc}
\hline & $\mathbf{n}$ & Shock & No shock & p \\
\hline Urinary tract occlusion & 54 & 19 & 35 & \\
No urinary tract occlusion & 23 & 12 & 11 & $<\mathbf{0 . 0 0 0 1}$ \\
Drainage & 34 & 22 & 12 & \\
No drainage & 43 & 9 & 34 & $\mathbf{0 . 0 0 3}$ \\
Double J ureteral stent & 25 & 16 & 9 & \\
Single J ureteral stent & 7 & 4 & 3 & \\
Pyelonephrostomy & 3 & 2 & 1 & \\
Ureterocutaneostomy & 1 & 0 & 1 & 0.6400 \\
\hline
\end{tabular}




\begin{tabular}{|c|c|c|c|}
\hline & Shock & No shock & p \\
\hline $\begin{array}{l}\text { White blood cell count, } \\
\text { average (range) }\left(/ \mathrm{mm}^{3}\right)\end{array}$ & $\begin{array}{c}18,816 \\
(3,100-54,670)\end{array}$ & $\begin{array}{c}13,728 \\
(3,300-46,460)\end{array}$ & 0.0236 \\
\hline $\begin{array}{l}\text { Platelet count, average } \\
\text { (range) }\left(\times 10^{3} / \mathrm{mm}^{3}\right)\end{array}$ & $\begin{array}{c}13.2 \\
(1.3-41.8)\end{array}$ & $\begin{array}{c}23.3 \\
(3.5-149.9)\end{array}$ & 0.0140 \\
\hline $\begin{array}{l}\text { C-reactive protein, } \\
\text { average (range) (mg/dl) }\end{array}$ & $\begin{array}{c}20.778 \\
(0.82-47.9)\end{array}$ & $\begin{array}{c}8.436 \\
(0.284-30.7)\end{array}$ & $<0.0001$ \\
\hline
\end{tabular}

occlusion. ${ }^{12}$ In those situations, shock due to urosepsis is the most dangerous and, therefore, the risk factors need to be clear to achieve early diagnosis and treatment. ${ }^{2}$ Risk factors may vary between institutions or hospitals due to different patient characteristics, so single-centre studies are somewhat limited. Our multicentre study demonstrated that high CRP is an independent risk factors for shock, especially in cases with urinary tract occlusion $(\mathrm{p}=0.0041)$.

CRP has been considered to reflect the onset of inflammation and bacterial infection, and Wang et al showed that elevated baseline high-sensitivity CRP was associated with increased risk of subsequent sepsis..$^{13}$ As to other inflammation marker, some studies show that serum procalcitonin has a role in more accurate prediction of bacterial infection ${ }^{14}$ and bacteremia with febrile UTI ${ }^{15}$ than CRP and WBC count.

Our data showed significantly more cases of shock with urinary tract occlusion that required stenting. Urosepsis cases involving urinary tract obstruction need prompt treatment to remove the causative infectious factors, such as a stone, for instance. ${ }^{2}$ Before and during treatment, urinary tract obstruction easily leads to bacterial invasion of the bloodstream, making rapid treatment necessary. ${ }^{16}$ Our data support this finding, but further prospective studies focusing on urinary tract occlusion cases are necessary for definitive conclusions.

As to the causative bacteria in bacteremia, E. coli was the most often isolated (34 strains, 44.2\%) and gram-negative bacteria occurred in the the majority $(70.7 \%)$ of our cases. Historically, the commonest isolates in uroseptic cases are gram-negative bacteria, such as E. coli or Pseudomonas aeruginosa $(70 \%)^{4,17}$ and our data support these studies. The antimicrobial susceptibilities of isolates from urosepsis show a recent trend of increases in ESBL-producing bacteria and fluoroquinolone-resistant bacteria..$^{18}$ Our data also showed high rates of fluoroquinolone (CPFX or LVFX) resistance $(37.0 \%)$ and ESBLs (25.9\%), reflecting the recent trend. As to the ESBL-producing bacteria, most (85.7\%) cases were fluoroquinolone-resistant bacteria as seen in the previous literature. ${ }^{19}$ Such resistant strains require long-term attention and monitoring.

Kadoya et al found that severe underlying disease, such as malignancy, medical use of a central vein line or drainage, older age, and drug use causing immune suppression (such as chemotherapy or steroid-dosing), were risk factors for bacteremia. ${ }^{20}$ Our series showed a high rate of malignancy, DM, and older patients, but no significant differences for shock. In

\begin{tabular}{|c|c|c|c|c|c|}
\hline Bacteria & $\mathbf{n}$ & Shock & Percent & No shock & Percent \\
\hline Escherichia coli & 34 & 18 & 54.5 & 16 & 28.1 \\
\hline ESBL-producer & 8 & 5 & 15.5 & 3 & 5.3 \\
\hline Klebsiella spp & 9 & 1 & 3.0 & 8 & 14.0 \\
\hline Pseudomonas aeruginosa & 6 & 1 & 3.0 & 5 & 8.8 \\
\hline MRSE & 6 & 0 & 0.0 & 6 & 10.5 \\
\hline S. aureus & 4 & 2 & 6.1 & 2 & 3.5 \\
\hline E. faecalis & 4 & 1 & 3.0 & 3 & 5.3 \\
\hline CNS & 3 & 1 & 3.0 & 2 & 3.5 \\
\hline P. mirabilis & 2 & 1 & 3.0 & 1 & 1.8 \\
\hline Achromobacter xylosoxidans & 2 & 0 & 0.0 & 2 & 3.5 \\
\hline MRSA & 2 & 0 & 0.0 & 2 & 3.5 \\
\hline E. cloacae & 2 & 1 & 3.0 & 1 & 1.8 \\
\hline C. koseri & 1 & 1 & 3.0 & 0 & 0.0 \\
\hline E.aerogenes & 1 & 0 & 0.0 & 1 & 1.8 \\
\hline E. cloacae complex (AmpC) & 1 & 1 & 3.0 & 0 & 0.0 \\
\hline Bacillus subtilis & 1 & 0 & 0.0 & 1 & 1.8 \\
\hline Micrococcus spp & 1 & 0 & 0.0 & 1 & 1.8 \\
\hline Paenibacillus urinalis & 1 & 0 & 0.0 & 1 & 1.8 \\
\hline Propionibacterium acnes & 1 & 0 & 0.0 & 1 & 1.8 \\
\hline S. agalactiae (B) & 1 & 0 & 0.0 & 1 & 1.8 \\
\hline Culture negative & 3 & 3 & 9.1 & 0 & 0.0 \\
\hline
\end{tabular}

ESBL: extended-spectrum beta lactamase; CNS: central nervous system; MRSA: methicillin-resistant S. aureus; MRSE: methicillin-resistent S.epidermidis 


\begin{tabular}{lccc}
\hline Table 5. Antibiotic susceptibilities of & E. coli & & \\
\hline & n & Shock & $\begin{array}{c}\text { No } \\
\text { shock }\end{array}$ \\
\hline ESBL & 7 & 4 & 3 \\
Non-ESBL & 20 & 8 & 12 \\
Fluoroquinolone-resistant & 10 & 5 & 5 \\
Fluoroquinolone-susceptible & 17 & 7 & 10 \\
ESBL + fluoroquinolone-resistant & 4 & 2 & 2 \\
Non-ESBL + fluoroquinolone-resistant & 6 & 4 & 2 \\
\hline ESBL: extended-spectrum beta lactamase. & \multicolumn{3}{c}{}
\end{tabular}

their cases with urosepsis, Oshida et al reported increases or decreases of serum WBC and no change or increase in CRP, ${ }^{21}$ but we demonstrated higher CRP and no change in WBC in uroseptic shock patients as risk factors for shock. Serum WBC is generally low in cases with severe sepsis, but our series had only two cases with decreased WBC $(\leq 4000 / \mu \mathrm{L})$ and only one case went into shock. On the other hand, 45 of our cases had high serum WBC $(\geq 12000 / \mu \mathrm{L})$ and $24(53.3$ $\%)$ of these went into shock. The difference in these findings could be based on the following: 1) shock due to urosepsis may be different from other kinds of shock; 2) our case series may possibly include more moderately severe cases compared with other studies because we had no cases leading to death; and 3) our cases were possibly diagnosed properly at a comparatively earlier stage and appropriate therapies

\begin{tabular}{lcc}
\hline Table 6. Antibiotic susceptibilities & \\
\hline Antibiotic & Shock & No shock \\
\hline Meropenem & 16 & 7 \\
Vancomycin & 1 & 1 \\
Ceftazidime & 2 & 1 \\
Cefmetazole & 4 & 6 \\
Ceftriaxone & 5 & 6 \\
Doripenem & 1 & \\
Tazobactam/ & 2 & 10 \\
piperacillin & & 4 \\
Ampicillin/sulbactam & & 3 \\
Cefepime & & 1 \\
Cefotaxime & & 5 \\
Cefozopran & & 1 \\
Daptomycin & & 1 \\
Clarithromycin & & 2 \\
Cefotiam & & 1 \\
No-use & 32 & 49 \\
Total & &
\end{tabular}

initiated sooner. Further studies with more patients need to be done to draw definitive conclusions, as mentioned above.

We also showed a significant difference in antibiotic usage between the cases with shock and without shock, suggesting that even though it cannot be known if antibiotic usage is the result or cause, MEPM was often used in the

\begin{tabular}{|c|c|c|c|c|c|c|}
\hline \multirow[t]{2}{*}{ Risk factor } & \multicolumn{3}{|c|}{ Univariate analysis } & \multicolumn{3}{|c|}{ Multivariate analysis } \\
\hline & HR & $95 \% \mathrm{Cl}$ & $\mathbf{p}$ & HR & $95 \% \mathrm{Cl}$ & p \\
\hline Sex (female) & 3.095 & $1.194-8.019$ & 0.0200 & 1.213 & $0.377-3.905$ & 0.7464 \\
\hline Age & 1.013 & $0.985-1.041$ & 0.3753 & & & \\
\hline Cancer & 0.260 & $0.085-0.794$ & 0.0181 & 0.500 & $0.132-1.894$ & 0.3077 \\
\hline Diabetes mellitus & 1.469 & $0.497-4.372$ & 0.4872 & & & \\
\hline Cerebral disease & 1.552 & $0.207-11.639$ & 0.6691 & & & \\
\hline Neurological disease & 0.750 & $0.065-8.646$ & 0.8176 & & & \\
\hline Leukocyte & 1.000 & $1.000-1.000$ & 0.0348 & 1.000 & $1.000-1.000$ & 0.9962 \\
\hline Platelet & 0.915 & $0.863-0.970$ & 0.0029 & 0.957 & 0.899-1.018 & 0.1604 \\
\hline C-reactive protein & 1.117 & $1.060-1.177$ & $<0.0001$ & 1.098 & $1.030-1.170$ & 0.0041 \\
\hline Urological obstruction & 0.484 & $0.180-1.301$ & 0.1502 & & & \\
\hline E. coli & 2.046 & $0.775-5.402$ & 0.1482 & & & \\
\hline E. coli (ESBL) & 2.821 & $0.622-12.785$ & 0.1787 & & & \\
\hline E. cloacae & 1.552 & $0.207-11.639$ & 0.6691 & & & \\
\hline E. faecalis & 0.489 & $0.049-4.928$ & 0.5438 & & & \\
\hline K. pneumoniae & 0.228 & $0.026-1.193$ & 0.1812 & & & \\
\hline$P$ aeruginosa & 0.280 & $0.031-2.521$ & 0.2562 & & & \\
\hline S. aureus & 0.489 & $0.049-4.928$ & 0.5438 & & & \\
\hline P. mirabillis & 1.553 & $0.092-25.468$ & 0.7656 & & & \\
\hline Ceftazidime resistance & 2.067 & $0.509-8.402$ & 0.3100 & & & \\
\hline Imipenem resistance & 0.394 & $0.076-2.037$ & 0.2665 & & & \\
\hline Levofloxacin resistance & 0.851 & $0.293-2.473$ & 0.7665 & & & \\
\hline
\end{tabular}

Cl: confidence interval; ESBL: extended-spectrum beta lactamase; HR: hazard ratio. 
cases with shock and TAZ/PIPC in those without; this finding may be evidence for urosepsis cases and shock.

We would like to emphasize the study limitations. First, a greater number of patients would provide more definitive evidence; second, this was a retrospective study, with all of the limitations that implies. Third, inflammation and/or infection-related markers or molecules, such as procalcitonin or lipopolysaccharide of outer membrane, were not examined. Fourth, we lacked the following data: the timing of measurements in relation to the risk factors, results of urine culture, details of the standard antimicrobial regimen, and strategy of drainage for obstructive uropathy. Fifth, matters concerning the urinary calculi and the reasons of urinary tract obstruction were unknown and the data related to shock or disseminated intravascular coagulation (DIC) are missing. Finally, the number of patients and study periods were unevenly distributed among the hospitals.

\section{Conclusion}

High CRP was identified as a risk factor for shock during urosepsis in our retrospective, multicentre analysis of data from a broad range of hospital patient characteristics. Further prospective studies with a greater number of patients are needed for definitive conclusions.

Competing interests: The authors report no competing personal or financial interests.

This paper has been peer-reviewed.

\section{References}

1. Shigemura K, Tanaka K, Adachi M, et al. Chronological change of antibiotic use and antibiotic resistance in Escherichia coli causing urinary tract infections. J Infect Chemother 2011;17:646-51. https://doi.org/10.1007/s10156-011-0241-2

2. Wagenhener FM, Pilatz A, Weidner W. Urosepsis from the view of the urologist. Int J Antimicrob Agents 2011;38:S51-7. https://doi.org/10.1016/i.jjantimicag.2011.09.007

3. Nicolle LE. Urinary tract pathogens in complicated infection and in elderly individuals. I Infect Dis 2001:183:S5-8. https://doi.org/10.1086/318844
4. Wagenlehner FM, Pilatz A, Naber KG, et al. Therapeutic challenges of urosepsis. Eur J Clin Invest 2008;38 S45-9. https://doi.org/10.1111/i.1365-2362.2008.02008.x

5. Wagenlehner FM, Naber KG. Hospital-acquired urinary tract infections. J Hosp Infect 2000;46:171-81. https://doi.org/10.1016/S0195-6701(00)90821-1

6. CLSI. Performance standards for antimicrobial susceptibility testing: 20th informational supplement. CLSI document M100-S19; Clinical and Laboratory Standards Institute, Wayne, PA. 2009.

7. Wagenlehner FM, Lichtenstern C, Rolfes $C$, et al. Diagnosis and management for urosepsis. Int I Urol 2013;20:963-70. htrps://doi.org/10.1111/iju.12200

8. Levy MM, Fink MP, MarshallIC, et al for the International Sepsis Definitions Conference 2001 SCCM/ ESICM/ACCP/ATS/SIS International Sepsis Definitions Conference. Intensive Care Med 2003;29:530-8.

9. Vincent JL. Clinical sepsis and septic shock-definition, diagnosis, and management principles. Langenbecks Arch Surg 2008;393:817-24. https://doi.org/10.1007/s00423-008-0343-1

10. Otto $\mathrm{G}$, Sandberg T, Marklund Bl, et al. Virulence factors and pap genotype in Escherichia coli isolates from women with acute pyelonephritis, with or without bacteremia. Clin Infect Dis 1993;17:448-56. https://doi.org/10.1093/dinids/17.3.448

11. Tandoğdu Z, Bartoletti R, Cai T, et al. Antimicrobial resistance in urosepsis: Outcomes from the multinational, multicentre global prevalence of infections in urology (GPIU) study 2003-2013. World I Urol 2016;34:1193-200. https://doi.org/10.1007/s00345-015-1722-1

12. Lee JH, Lee YM, Cho JH. Risk factors of septic shock in bacteremic acute pyelonephritis patients admitted to an ER. J Infect Chemother 2012;18:130-3. https://doi.org/10.1007/s10156-011-0289-z

13. Wang HE, Shapiro NI, Safford MM, et al. High-sensitivity C-reactive protein and risk of sepsis. PLoS One 2013;8:e69232. https://doi.org/10.1371/journal.pone.0069232

14. Simon L, Gauvin F, Amre DK, et al. Serum procalcitonin and C-reactive protein levels as markers of bacterial infection: A systematic review and meta-analysis. Clin Infect Dis 2004;15:206-17. https://doi.org/10.1086/421997

15. van Nieuwkoop C, Bonten TN, van'† Wout JW, et al. Procalcitonin reflects bacteremia and bacterial load in urosepsis syndrome: A prospective observational study. Crit Care 2010;14:R206. https://doi.org/10.1186/cc9328

16. Gutierrez J, Smith A, Geavlete $P$, et al. Urinary tract infections and postoperative fever in percutaneous nephrolithotomy. World J Urol 2013;31:1135-40. https://doi.org/10.1007/s00345-012-0836-y

17. Kalra OP, Raizada A. Approach to a patient with urosepsis. J Glob Infect Dis 2009;1:57-63. https://doi.org/10.4103/0974-777X.52984

18. Lee JC, Lee NY, Lee HC, et al. Clinical characteristics of urosepsis caused by extended-spectrum betalactamase-producing Escherichia coli or Klebsiella pneumonia and their emergence in the community. J Microbiol Immunol Infect 2012;45:127-33. https://doi.org/10.1016/i.mii.2011.09.029

19. Yoshikawa K, Moritake J, Suzuki K, et al. Prevalence and drug-susceptibilities of extended-spectrum -lactamase producing Escherichia coli strains isolated from urine. Jpn J Chemother 2014;62:198-203.

20. Kadoya $M$, Nada $T$, lida $E$, et al. [Bacterial survey of patients with bacteremia during the ten years (1978-1987) in Nagoya University Hospital]. Kansenshogaku Zasshi 1991;65:168-74.

21. Oshida $\mathrm{Y}$, Hirashima 0 , Tanaka $\mathrm{T}$, et al. [The characteristics of urinary tract infection with urosepsis]. Kansenshogaku Zasshi 2014;88:678-84.

Correspondence: Dr. Katsumi Shigemura, Division of Urology, Department of Organ Therapeutics, Faculty of Medicine, Kobe University Graduate School of Medicine, Kobe, Japan; katsumi@med.kobe-u.ac.jp 